ARTICLE

Received 19 Apr 2015 | Accepted 9 Jun 2015 | Published 16 Jul 2015

DOI: $10.1038 /$ ncomms 8767

OPEN

\title{
A spectrally tunable all-graphene-based flexible field-effect light-emitting device
}

\author{
Xiaomu Wang ${ }^{1,2, \star, \dagger}$, He Tian ${ }^{1,2, \star}$, Mohammad Ali Mohammad ${ }^{1,2}$, Cheng $\mathrm{Li}^{1,2}$, Can Wu ${ }^{1,2}$, Yi Yang ${ }^{1,2}$ \\ \& Tian-Ling $\operatorname{Ren}^{1,2}$
}

The continuous tuning of the emission spectrum of a single light-emitting diode (LED) by an external electrical bias is of great technological significance as a crucial property in highquality displays, yet this capability has not been demonstrated in existing LEDs. Graphene, a tunable optical platform, is a promising medium to achieve this goal. Here we demonstrate a bright spectrally tunable electroluminescence from blue $(\sim 450 \mathrm{~nm})$ to red $(\sim 750 \mathrm{~nm})$ at the graphene oxide/reduced-graphene oxide interface. We explain the electroluminescence results from the recombination of Poole-Frenkel emission ionized electrons at the localized energy levels arising from semi-reduced graphene oxide, and holes from the top of the $\pi$ band. Tuning of the emission wavelength is achieved by gate modulation of the participating localized energy levels. Our demonstration of current-driven tunable LEDs not only represents a method for emission wavelength tuning but also may find applications in high-quality displays.

\footnotetext{
${ }^{1}$ Institute of Microelectronics, Tsinghua University, Beijing 100084, China. ${ }^{2}$ Tsinghua National Laboratory for Information Science and Technology (TNList), Tsinghua University, Beijing 100084, China. * These authors contributed equally to this work. † Present address: Department of Electrical Engineering, Yale University, New Haven, Connecticut 06511, USA. Correspondence and requests for materials should be addressed to T.-L.R. (email: RenTL@tsinghua.edu.cn).
} 
T he solid-state light-emitting diode (LED) is a key component of today's semiconductor industry ${ }^{1}$. The successful development of LEDs has enabled plenty of applications such as high-performance communication, low-cost lighting and smart displays. In modern LED industries, controlling the colour of LEDs is a challenging task ${ }^{2-6}$. Traditional LEDs are available in different predefined colours whose emission wavelength is adjusted by complex material design and bandgap engineering. To date, the in situ controlling of colour in a single device has never been realized, although it is highly desired. Graphene and its derivative material-based photonic devices are currently the focus of intense study ${ }^{7-12}$. Graphene is an amendable platform whose electronic and optical properties can be tailored by chemical and electrical means. This property is particularly interesting as it potentially provides a way to in situ control the colour of LEDs. Although various high-performance graphene-based photonic devices have been reported ${ }^{13-17}$, unfortunately the development of graphene-based LEDs has been unsuccessful owing to its vanishing bandgap.

A plausible strategy for realizing electroluminescence (EL) may be by creating discrete energy levels by utilizing functional groups, quantum confinement effect or other mid gap states. Unfortunately, the insulating nature of the photoluminescent graphene derivatives, such as functionalized graphene or solution-based graphene quantum dots, negatively affects the carrier injection and disables EL. In summary, a method to fabricate a graphene-based device with a non-vanishing bandgap and charge injection capability is still lacking.

In this article, we demonstrate a desirable combination of a bandgap structure and a bipolar carrier injection in a special type of semi-reduced graphene oxide (GO). Our device formed on a laser-scribed GO surface consists of a series of rGO nanoclusters with many different sizes, which can selectively stimulate a single-colour luminescence by controlling its doping level. The semi-reduced GO network is with a mobility approaches $10 \mathrm{~cm}^{2} \mathrm{~V}^{-1} \mathrm{~s}^{-1}$, which supports the carrier injection. We report the observation of EL in these semi-reduced GO-based devices. The light-emission spectrum in our device is in situ adjusted from blue $(\lambda \sim 450 \mathrm{~nm})$ to red $(\lambda \sim 750 \mathrm{~nm})$ by electrical gating or conditioning the environmental doping. The device shows a high brightness of up to $6,000 \mathrm{~cd} \mathrm{~m}^{-2}$, with efficiency around $1 \%$.

\section{Results}

Fabrication and characterization of field-effect LED. Figure 1a schematically shows the structure of our all-graphene-based fieldeffect LED (GFLED). A planar side gate graphene field-effect transistor device was first prepared based on a laser-scribing method (see Methods for details) ${ }^{18}$. The light-emitting layer, which is the interface between the $\mathrm{GO}$ and $\mathrm{rGO}$, was then uncovered by using current annealing to remove the highly conductive rGO channel. The typical length of the light-emitting region is $80-120 \mu \mathrm{m}$ and is located in the centre of the narrow graphene field-effect transistor channel. This central location is determined by the geometric design as the Joule heating rapidly affects the narrowest part of the structure. It is worth mentioning here that the fabrication process was carried out in ambient conditions and does not require any high-vacuum environment, hazardous solution or high-temperature treatment.

The exposed interfacial layer obtained by this method presents three major differences as compared with GO or rGO. First, this interfacial layer is ultra-flat. The RMS thickness obtained from atomic force microscope is several orders smaller than that of GO or rGO. Second, the interfacial layer is colourful, this is in stark contrast to the white colour of GO and the black colour of rGO. Third, and perhaps most importantly, a strong and broadband photoluminescence is solely observed in the interfacial layer, as shown in Fig. 1b. (See Supplementary Figs 1-4 and Supplementary Note 1 for detailed comparison between the interfacial layer and bulk GO/rGO.) The photo-luminescence (PL) spectra of the GO/rGO interfacial layer were obtained by exciting the samples with a continuous wave $457 \mathrm{~nm}$ laser with low excitation power $(\sim 100 \mu \mathrm{W})$. The interfacial layer showed very broad PL, ranging from about 470 to more than $720 \mathrm{~nm}$ (with $\sim 0.8 \mathrm{eV}$ full-width at half-maximum).

Generally speaking, the zero-bandgap nature of rGO results in a large non-radiative decay rate of stimulated electron-hole pairs, while the energy gap between the $\pi$ and $\pi^{*}$ bands of GO is extremely large. All these factors imply that luminescence in rGO and GO should be impossible. The experimentally observed broad PL feature is a strong signature of the presence of localized states, similar as those in rGO quantum dots ${ }^{19-20}$. These localized states can be understood by the formation of a transition state during the reduction process: there is a distinct partially reduced GO layer between the GO precursor and the thermally induced rGO as a result of the laser dosage gradient from the surface downwards.

We performed X-ray photoemission spectroscopy to further characterize the chemical composition of the light-emitting layer. A collection of X-ray photoemission spectroscopy spectra is shown in Fig. 1c. Fundamentally, the light-emitting layer can be identified as an intermediate state between GO and rGO in terms of oxygen concentration. The $\mathrm{C} / \mathrm{O}$ ratio for $\mathrm{rGO}$, the interface layer and GO is $2.10,0.77$ and 0.57 , respectively. The chemical states of carbon and oxygen in different layers also show pronounced variation. The $\mathrm{C} 1 \mathrm{~s}$ spectra of $\mathrm{GO}$ and $\mathrm{rGO}$ are similar as per the previously reported typical results ${ }^{21}$. For the GO case, in addition to a large carbon peak composed of graphitic $s p^{2}$ and $s p^{3} \mathrm{C}-\mathrm{C}$ bonds, another $\mathrm{C}-\mathrm{O}$ clear peak can be observed due to the hydroxyls and epoxies. For the rGO case, the spectrum is dominated by the graphitic $s p^{2}$ component and the C-O bonds only show a very minute component. However, the interfacial layer exhibits distinct features as compared with the GO and rGO. In addition to an intermediate carbon-carbon bond state, the interfacial layer is also rich in $\mathrm{C}=\mathrm{O}$ bonds. These bonds are usually attributed to carbonyls. The presence of carbonyls in the GO basal plane signifies incomplete reduction ${ }^{21}$. A similar feature of the transition state was also observed in the $\mathrm{O} 1 \mathrm{~s}$ spectra. While $\mathrm{rGO}$ and $\mathrm{GO}$ are dominated by $\mathrm{C}=\mathrm{O}$ and $\mathrm{C}-\mathrm{O}$ peaks, respectively, the light-emitting layer is a mixture of $\mathrm{C}-\mathrm{O}$ and $\mathrm{C}=\mathrm{O}$ bonds. The two-component configuration has been used to characterize the transition state in the evolution of reduction of $\mathrm{GO}^{22}$.

As described above, the interfacial layer is a special type of partially reduced GO. Generally speaking, the reduction of GO leads to creation of small $(2-3 \mathrm{~nm})$ ordered $s p^{2}$ clusters isolated within the $s p^{3} \mathrm{C}-\mathrm{O}$ matrix of $\mathrm{GO}^{7}$. Therefore, the partially reduced GO likely results in a series of discrete energy levels between $\pi$ and $\pi^{\star}$ bands by quantum confinement effect ${ }^{19}$. Incident laser stimulates electron-hole pairs in the $\pi$ band and in these discrete states. Radiative recombination of the electron pairs thus gives rise to a PL effect (and also leads to EL, see discussion below). In this case, the degree of reduction of the rGO determines the features of the PL spectrum, similar to how porous silicon and colloidal quantum dot devices affect the spectrum $^{6,23}$.

Spectrally adjustable EL. Once the energy levels with bandgaps are created, EL can be expected if bipolar carriers are injected into the device. Previously reported 'gapped graphene' (such as GO and graphene quantum dots) is either an insulator or a liquidphase material, which impair the carrier injection. Fortunately, the interfacial layer (although also a type of GO), is a p-type 
a
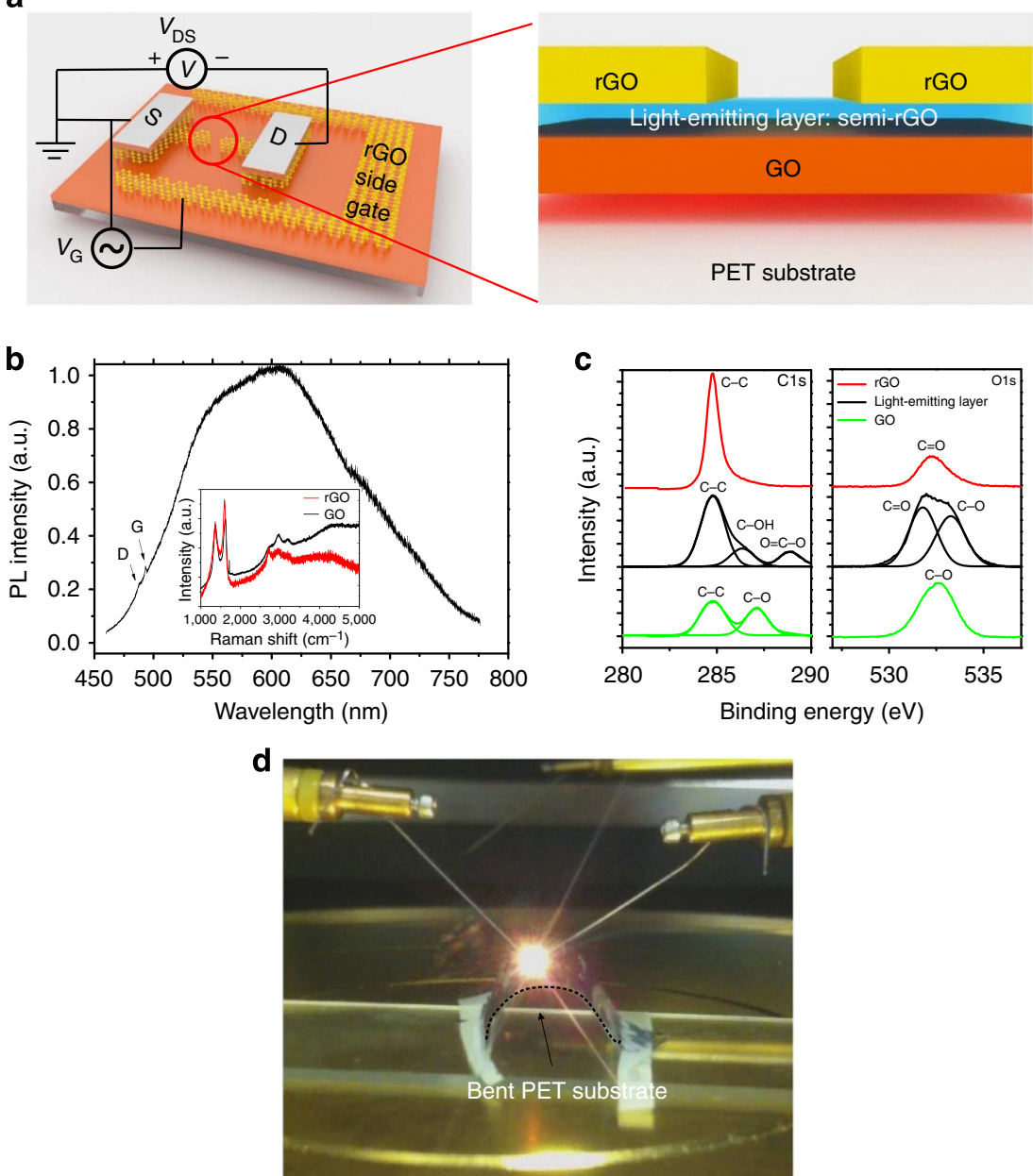

Figure 1 | GFLED and characterization. (a) Schematic of the GFLED. A distinct semi-reduced GO (blue) at the interface between GO (orange) and rGO (gold) is responsible for light emission. (b) PL spectrum of light-emitting layer. The D and G Raman peaks are marked. Inset: Raman spectra of the GO and rGO samples. The GO and rGO samples do not show any PL signals. (c) X-ray photoemission spectroscopy spectra of rGO (red), GO (green) and the lightemission layer (black). Spectra from the $\mathrm{C}$ 1s and $\mathrm{O}$ 1s orbitals are shown. (d) Bright red light emission from the GFLED on a flexible polyethylene terephthalate (PET) substrate under a $12 \mathrm{~V}$ bias voltage and a $0.1 \mathrm{~A}$ drive current. The GFLED size is around $100 \times 100 \mu \mathrm{m}$. The edge of the bent PET is marked by a dashed line. The bending radius is $\sim 8 \mathrm{~mm}$.

semiconductor with a good conductivity (Fig. 2a inset; also see Supplementary Figs 5-7 and Supplementary Note 2 for characterization of its field effect). In addition, an enhanced carrier injection was also identified under a strong bias field, manifested by the rapidly increasing current versus bias voltage in Fig. 2a. Accompanied by the pronounced free carrier injection process in the device, we observed EL along the interfacial layer. The emitted light is bright and easily identified by the naked eye (see Fig. 1d). We fabricated 20 devices to characterize the bias-dependent EL intensity. Typical relative emission intensity versus different drain bias and the EL intensity distribution are plotted in Fig. 2a,b, respectively. Notably, the EL intensity is controlled by drive current. As shown in Fig. 2b, the devices are relatively uniform under a fixed $0.1 \mathrm{~A}$ current bias $\left(I_{\max } / I_{\min }<2\right)$. In contrast, the relative EL intensity presents large device to device variation $\left(I_{\max } / I_{\min } \sim 10\right)$ under a fixed $12 \mathrm{~V}$ voltage.

An exceptional observation in this work is that the colour of EL emission can be continuously tuned from light blue to dark red by adjusting the Fermi levels. The Fermi level and the doping level of the GFLED can be modulated either electrically or chemically. In this paper, we use a side gate field to control the Fermi level; however, the colour also changes in response to ambient doping, enabling sensing applications. Typical emission images, spectra recorded at different side gate voltages during the EL measurement and spatially resolved EL are shown in Figs $1 \mathrm{~d}$ and $2 \mathrm{c}$ and Supplementary Fig. 8. The EL peaks exhibit sharp single Lorentzian shapes. As shown in Fig. 2c, the peak emission wavelength shifts from $690 \mathrm{~nm}$ at zero gate voltage to $470 \mathrm{~nm}$ under $50 \mathrm{~V}$ gate voltage. On one hand, the EL peaks present similar narrow full-width at half-maximum $(\sim 0.2 \mathrm{eV})$, whereas on the other hand, the relative intensity of the EL is found to vary under different bias voltages. For instance, the maximum luminance and the luminous efficacy of the blue light are much lower than the red light. Approximately $4.8 \mathrm{~lm} \mathrm{~W}^{-1}$ (corresponding to $\sim 0.7 \%$ external quantum efficiency, also see Supplementary Figs 9 and 10 for details) and $6,000 \mathrm{~cd} \mathrm{~m}^{-2}$ red and green lights were measured under a $12 \mathrm{~V}$ bias and a $0.1 \mathrm{~A}$ drive current, whereas $0.67 \mathrm{~lm} \mathrm{~W}^{-1}$ (corresponding to $\sim 0.1 \%$ external quantum efficiency) and $800 \mathrm{~cd} \mathrm{~m}^{-2}$ blue light was measured under a $16.5 \mathrm{~V}$ bias and a $0.1 \mathrm{~A}$ drive current. Overall, the broad PL can be roughly regarded as the envelope of all these possible EL spectra. This strongly suggests that the EL results from a selective excitation of inhomogeneous luminescence of semi-reduced GO, as observed in previously reported $\mathrm{rGO}$ quantum dots. A possible explanation of the field effect-modified EL will be discussed in the following part. 

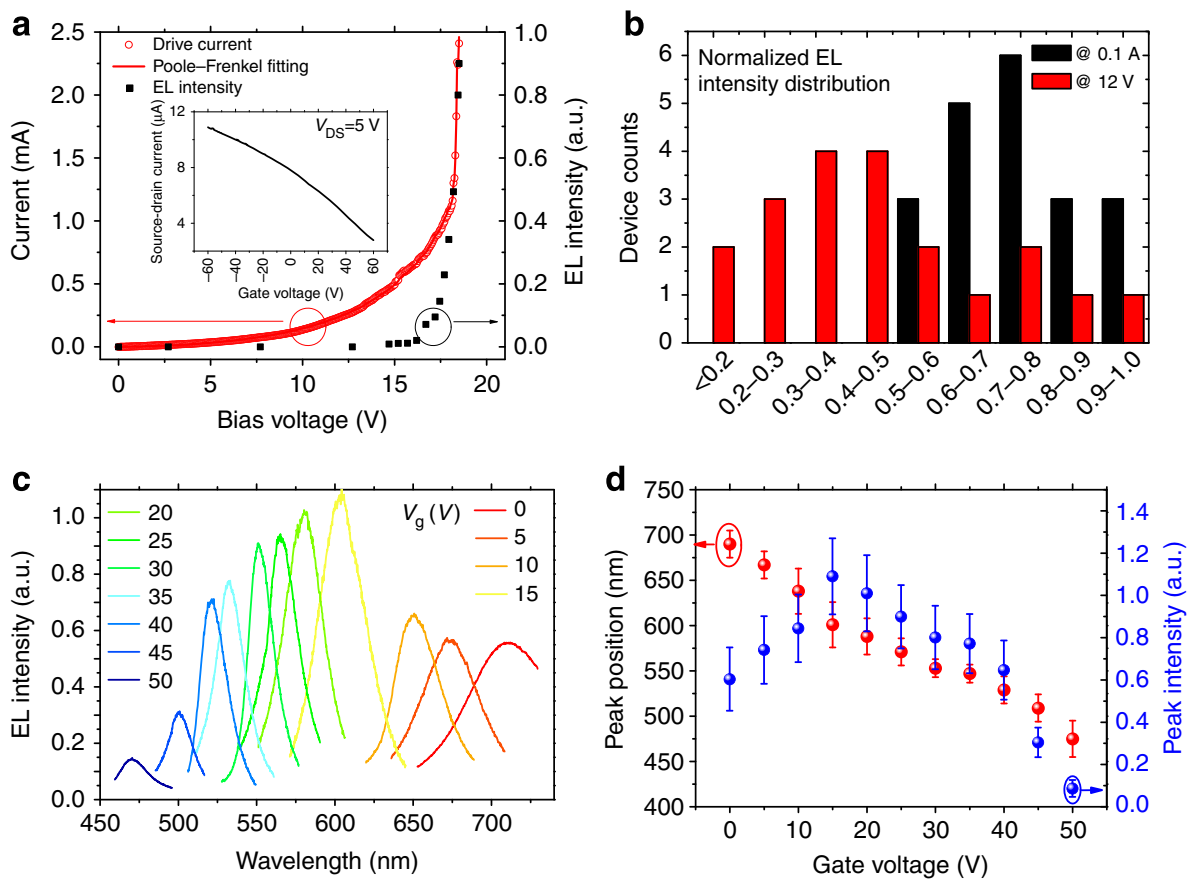

Figure 2 | Electroluminescence of GFLED. (a) Source-drain current (circles) and EL intensity (solid squares) versus source-drain bias voltage. The device used has a W/L ratio around $0.1 \mathrm{~mm} / 0.1 \mathrm{~mm}$. The solid line shows the Poole-Frenkel fitting of the drive current. The EL peak is at $690 \mathrm{~nm}$. Inset: source-drain current versus gate voltage of the same device, showing a p-type field effect. (b) Histogram of EL intensity distribution under fixed voltage and current bias. The data have been obtained from 20 devices. (c) Typical EL spectra of a single GFLED. Gate biases are from 0 to 50 V. (d) Peak position (wavelength) and intensity of the GFLED EL versus gate voltage. The data have been collected from 19 devices. Error bars (standard uncertainty) are estimated from the device variations.

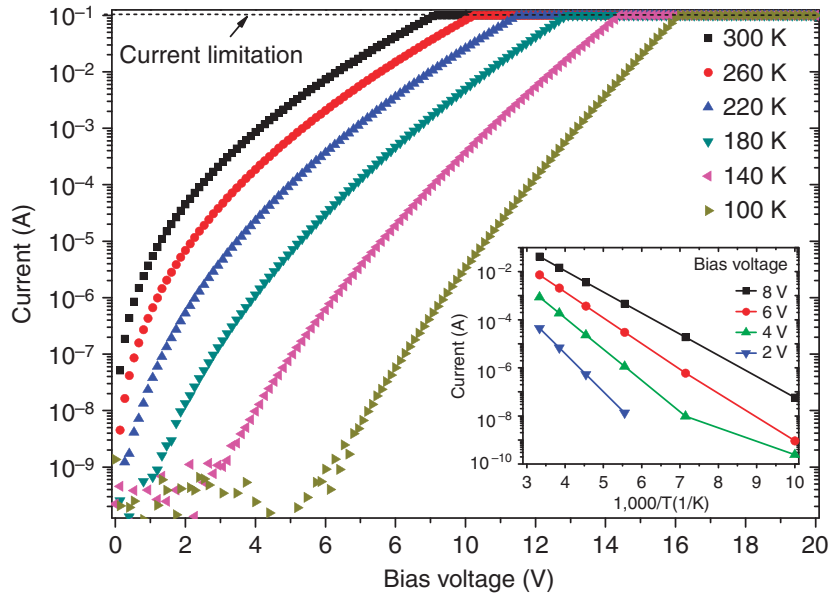

Figure 3 | GFLED two-terminal temperature-dependent transport.

Source-drain current versus voltage bias at different temperatures. The current is limited to $0.1 \mathrm{~A}$ to avoid device breakdown. Inset: Arrhenius plot of source-drain current at bias voltages of $2,4,6$ and $8 \mathrm{~V}$.

Currently, the efficiency of GFLEDs is relatively low. We hypothesize that two factors limit the efficiency. First, as shown in Supplementary Fig. 9, the efficiency tends to saturate at a large current density. We attribute this saturation to the inefficient injection of electrons caused by heat dissipation, that is, much of the supplied power is lost to heat dissipation. In other words, the saturation is caused by the inefficient injection of electrons (discussed below). The large amount of heat dissipated can also break the devices rapidly. The emission lifetime of these devices are several tens of seconds in ambient conditions. This short lifetime can be attributed to vigorous oxidation in air. However,

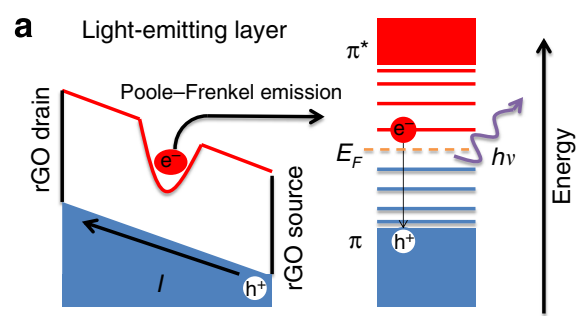

b

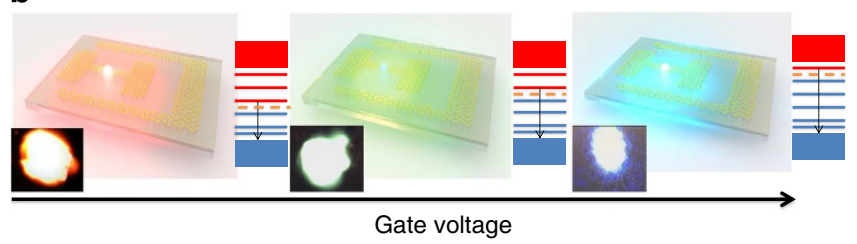

Figure 4 | GFLED light-emission mechanism. (a) A schematic of the charge injection process. The trapped electrons are excited to the lowest unoccupied discrete energy level by Poole-Frenkel emission. And the excited electron recombines with the hole in the $\pi$ band, resulting in photon emission. (b) Schematic of the gate voltage-dependent EL. The Fermi level determines the lowest unoccupied energy state that mainly participates in the radiative recombination. Inset: corresponding emission images from a real device.

these devices have a much longer half-life in vacuum and tests have revealed continuous and stable emission for up to $2 \mathrm{~h}$, suggesting protective coatings may help to avoid structural damage in practical devices. Second, in the absence of an intersystem cross path, only singlet excitons can be harvested, which suggests that the efficiency upper limit of GFLED is $25 \%$. Therefore, the promotion of efficiency requires improved 
carrier injection and introducing intersystem crossing in future works.

EL mechanism. Next, we proceed to discuss the possible EL mechanism. The simultaneously rapid increase of the current and EL intensity clearly indicates that carrier injection plays a central role in the EL process. Owing to the p-type nature of the light-emitting layer, electron injection is the key factor of understanding the EL process. At the current state, the exact mechanism of electron injection is not well known. On the basis of the various evidences, we hypothesize that the electric field-assisted thermal ionization, also known as Poole-Frenkel emission, is the most probable mechanism.

We performed temperature-dependent electrical measurements to investigate the electron injection process. Figure $4 \mathrm{a}$ shows the Poole-Frenkel plot of $I-V$ characteristics of a typical device. As illustrated in the inset of Fig. 3, a linear relationship fits well to the measured conductivity in the Arrhenius plot, indicating the electrons inject into the system on a charge emission manner. The highly linear feature in the $\log (I)$ versus $V^{1 / 2}$ plot signifies the $I-V$ curves agree well with the PooleFrenkel current law (see Supplementary Note 3 for details) ${ }^{24}$ The Poole-Frenkel effect is usually understood as a means by which trapped electrons get out of Coulomb binding and move to the conduction band. Figure 4a schematically shows how this effect applies to the GFLED. The oxide-rich light-emitting layer has a high density of electron trap states. Under high field, the localized electrons are excited into the lowest unoccupied discrete energy level. Radiative recombination between the thermally emitted electrons and the free holes results in the EL.

This mechanism also explains the observed spectral shift, as shown in Fig. 4b. Gating graphene lifts up the chemical potential and thus the energy level of the lowest unoccupied discrete state; therefore, the excited electron energy is increased. Through this way, the EL peak can be adjusted within the whole PL range; and the relative emission intensity is determined by the distribution of density of states of the discrete energy levels.

Several mechanisms, such as thermal emission ${ }^{25}$, FowlerNordheim tunnelling ${ }^{26,27}$ and impact excitations ${ }^{28-30}$ may also cause the EL in the GFLED. However, none of them can consistently explain all the findings of this work. Here we discuss the validity of these possibilities to GFLEDs. First of all, the featureless electroluminescence peak would correspond to a local temperature above $4,500 \mathrm{~K}$ if the EL results from blackbody radiation coming from Joule heating. This would rule out thermal emission as the light-emitting mechanism. Second, Fowler-Nordheim tunnelling, which is a main charge injection mechanism in porous silicon EL device, may also work for the GFLED $^{26}$. Considering the planar device structure, the tunnelling process most likely occurs around the drain electrode that holds the largest band bending. However, this contradicts the light emission observed in the middle of the device. Finally, impact excitation/ionization is another plausible EL mechanism and has been used to successfully describe the EL phenomenon in carbon nanotubes ${ }^{28-30}$. However, the threshold voltage in an impact ionization process is always accompanied by a negative temperature coefficient. Therefore, the observed positive temperature coefficient clearly rules out the impact ionization mechanism.

\section{Discussion}

In summary, we have reported the design and implementation of a LED device based on a special type of semi-reduced GO that forms in the $\mathrm{rGO} / \mathrm{GO}$ interface. By yielding a series of discrete energy levels between the $\pi-\pi^{\star}$ gap and injecting free carriers into those states, EL is successfully achieved. The in situ tuned wavelength of the EL exemplifies a kind of novel light-emitting device that is highly desired for high-color-quality displays and high-quality lightings. The precise spectral tunability, compact device structure, high performance and straightforward fabrication point to the commercial prospects of such a device. We anticipate our findings to be a prospective point for commercialization of graphene devices. Furthermore, the observance of EL itself addresses the lack of a light source component in graphene-based photonic devices, paving the way for all-graphene integrated photonics. This device opens the possibility of fabricating carbon-based photonic devices due to the henceforth availability of graphene-based LEDs.

\section{Methods}

Sample preparation. GFLEDs were fabricated based on a side gate field-effect transistor structure. An $\sim 1 \mu \mathrm{m}$ thick GO (XFNANO Materials Tech) film was prepared by drop casting on a flexible polyethylene terephthalate substrate. Conductive rGO traces with $\sim 100 \mu$ m width were then patterned by a previously reported laser-scribing method within a DVD drive ${ }^{31}$. Silver paste electrodes were fabricated to improve the contact. In the scribing process, the DVD drive's $788 \mathrm{~nm}$ laser $(5 \mathrm{~mW})$ was used to gradually reduce the stacked GO films to rGO. After that, the as-prepared devices were annealed by a $10 \mathrm{~mA}$ current to expose the interfacial layer.

Electrical measurement. Electrical measurements were carried out within a Lakeshore CPX-VF-457 cryogenic probe station using a Keithley 4200 semiconductor analyser. Electrical doping were achieved by using a side gate that surrounds the GFLED. The GO acts as a dielectric with a capacitance of $160 \mathrm{nF}$ (see Supplementary Fig. 5 for details).

PL and EL measurements. The luminescence measurements were performed using a Renishaw inVia micro-Raman spectroscope equipped with 457, 514 and $633 \mathrm{~nm}$ lasers. The spot diameter was around $1 \mu \mathrm{m}$ under a $\times 100$ objective lens. For PL measurements, the laser powers was set at $100 \mu \mathrm{W}$ to avoid breaking the samples. The typical integrating time was $10 \mathrm{~s}$. For EL measurements, a dualchannel Keithley 2612 source meter supplied the electrical and gate bias.

\section{References}

1. Zheludev, N. The life and times of the LED-a 100-year history. Nat. Photon. 1 , 189-192 (2007).

2. Koshida, N. \& Koyama, H. Visible electroluminescence from porous silicon. Appl. Phys. Lett. 60, 347-349 (1992).

3. Ponce, F. A. \& Bour, D. P. Nitride-based semiconductors for blue and green light-emitting devices. Nature 386, 351-359 (1997).

4. Friend, R. H. et al. Electroluminescence in conjugated polymers. Nature 397, 121-128 (1999).

5. Avouris, P., Freitag, M. \& Perebeinos, V. Carbon-nanotube photonics and optoelectronics. Nat. Photon. 2, 341-350 (2008).

6. Shirasaki, Y., Supran, G. J., Bawendi, M. G. \& Bulovic, V. Emergence of colloidal quantum-dot light-emitting technologies. Nat. Photon. 7, 13-23 (2013).

7. Loh, K. P., Bao, Q., Eda, G. \& Chhowalla, M. Graphene oxide as a chemically tunable platform for optical applications. Nat. Chem. 2, 1015-1024 (2010).

8. Ju, L. et al. Graphene plasmonics for tunable terahertz metamaterials. Nat. Nanotechnol. 6, 630-634 (2011).

9. Chen, J. et al. Optical nano-imaging of gate-tunable graphene plasmons. Nature 487, 77-81 (2012).

10. Fei, Z. et al. Gate-tuning of graphene plasmons revealed by infrared nanoimaging. Nature 487, 82-85 (2012).

11. Yan, H. et al. Tunable infrared plasmonic devices using graphene/insulator stacks. Nat. Nanotechnol. 7, 330-334 (2012).

12. Geim, A. K. \& Novoselov, K. S. The rise of graphene. Nat. Mater. 6, 183-191 (2007).

13. Xia, F., Mueller, T., Lin, Y.-M., Valdes-Garcia, A. \& Avouris, P. Ultrafast graphene photodetector. Nat. Nanotechnol. 4, 839-843 (2009).

14. Mueller, T., Xia, F. \& Avouris, P. Graphene photodetectors for high-speed optical communications. Nat. Photon. 4, 297-301 (2010).

15. Liu, M. et al. A graphene-based broadband optical modulator. Nature 474, 64-67 (2011).

16. Bao, Q. et al. Broadband graphene polarizer. Nat. Photon. 5, 411-415 (2011).

17. Wang, X., Cheng, Z., Xu, K., Tsang, H. K. \& Xu, J.-B. High-responsivity graphene/silicon-heterostructure waveguide photodetectors. Nat. Photon. 7, 888-891 (2013). 
18. El-Kady, M. F., Strong, V., Dubin, S. \& Kaner, R. B. Laser scribing of high-performance and flexible graphene-based electrochemical capacitors. Science 335, 1326-1330 (2012).

19. Eda, G. et al. Blue photoluminescence from chemically derived graphene oxide. Adv. Mater. 22, 505-509 (2010).

20. Chien, C.-T. et al. Tunable photoluminescence from graphene oxide. Angew. Chem. Int. Ed. 51, 6662-6666 (2012).

21. Gao, W., Alemany, L. B., Ci, L. \& Ajayan, P. M. New insights into the structure and reduction of graphite oxide. Nat. Chem. 1, 403-408 (2009).

22. Bagri, A. et al. Structural evolution during the reduction of chemically derived graphene oxide. Nat. Chem. 2, 581-587 (2010).

23. Bsiesy, A. et al. Voltage-controlled spectral shift of porous silicon electroluminescence. Phys. Rev. Lett. 71, 637-640 (1993).

24. Yeargan, J. R. \& Taylor, H. L. The Poole-Frenkel effect with compensation present. J. Appl. Phys. 39, 5600-5604 (1968).

25. Mann, D. et al. Electrically driven thermal light emission from individual single-walled carbon nanotubes. Nat. Nanotechnol. 2, 33-38 (2007).

26. Walters, R. J., Bourianoff, G. I. \& Atwater, H. A. Field-effect electroluminescence in silicon nanocrystals. Nat. Mater. 4, 143-146 (2005).

27. Adam, E. et al. Electroluminescence from single-wall carbon nanotube network transistors. Nano Lett. 8, 2351-2355 (2008).

28. Misewich, J. A. et al. Electrically induced optical emission from a carbon nanotube FET. Science 300, 783-786 (2003).

29. Chen, J. et al. Bright infrared emission from electrically induced excitons in carbon nanotubes. Science 310, 1171-1174 (2005).

30. Marty, L. et al. Exciton formation and annihilation during 1D impact excitation of carbon nanotubes. Phys. Rev. Lett. 96, 136803 (2006).

31. Tian, H. et al. Wafer-scale integration of graphene-based electronic, optoelectronic and electroacoustic devices. Sci. Rep. 4, 3598 (2014).

\section{Acknowledgements}

This work was supported by National Natural Science Foundation (61434001), 973

Program(2015CB352100), National Key Project of Science and Technology
(2011ZX02403-002) and Special Fund for Agroscientic Research in the Public Interest (201303107) of China. We are thankful for receiving support through the State Key Laboratory of Automotive Safety and Energy, Tsinghua University. H.T. is thankful for receiving support through the Ministry of Education Scholarship of China. M.A.M. is thankful for being supported by the postdoctoral fellowship (PDF) programme of the Natural Sciences and Engineering Research Council of Canada (NSERC).

\section{Author contributions}

X.W. and H.T. conceived the project and contributed equally to the sample preparation, characterization, device fabrication and EL measurements. X.W., H.T., M.A.M. and T.-L.R. analysed the data and wrote the paper; M.A.M., C.L., C.W. and Y.Y. assisted in the device fabrication; T.-L.R. supervised the project.

\section{Additional information}

Supplementary Information accompanies this paper at http://www.nature.com/ naturecommunications

Competing financial interests: The authors declare no competing financial interests.

Reprints and permission information is available online at http://npg.nature.com/ reprintsandpermissions/

How to cite this article: Wang, X. et al. A spectrally tunable all-graphene-based flexible field-effect light-emitting device. Nat. Commun. 6:7767 doi: 10.1038/ncomms8767 (2015).

(c) (i) This work is licensed under a Creative Commons Attribution 4.0 International License. The images or other third party material in this article are included in the article's Creative Commons license, unless indicated otherwise in the credit line; if the material is not included under the Creative Commons license, users will need to obtain permission from the license holder to reproduce the material To view a copy of this license, visit http://creativecommons.org/licenses/by/4.0/ 